\title{
A Case of a Concurrent and Co-Located Invasive Carcinoma and a Fibroadenoma to Illustrate the Potential of Dual-Energy, Contrast-Enhanced Digital Mammography on the Diagnosis of Complex Breast Lesions
}

\author{
Maria Del Mar Travieso Aja, ${ }^{1, *}$ Purificacion Munoz, ${ }^{1}$ Mario Rodriguez Rodriguez, ${ }^{1}$ Victor Vega Benitez, ${ }^{2}$ \\ and Octavio P. Luzardo ${ }^{3}$ \\ ${ }^{1}$ Imaging Diagnostic Service, San Roque Hospital Group, Las Palmas, Spain \\ ${ }^{2}$ Department of Surgery, Insular and Maternal and Child Hospitals (CHUIMI), Las Palmas, Spain \\ ${ }^{3}$ Research Group in Environment and Health, Research Institute of Biomedical and Health Sciences (IUIBS), Universidad de Las Palmas de Gran Canaria, Las Palmas, Spain \\ "Corresponding author: Maria Del Mar Travieso Aja, Imaging Diagnostic Service, San Roque Hospital Group, Las Palmas, Spain. Tel: +34-928012600, Fax: +34-928451461, E-mail: \\ marimartravieso@gmail.com
}

Received 2015 August 07; Revised 2015 September 16; Accepted 2015 December 26.

\begin{abstract}
Up to $19 \%$ of breast malignancies may be missed by conventional imaging techniques, especially when they are concurrent or colocated with other benign lesions. However, more sensitive techniques, such as magnetic resonance imaging (MRI), are often too expensive for routine use in developing countries. Contrast-enhanced, dual-energy digital mammography (CESM) is a recently introduced imaging modality whose performance has been reported to be similar to that of MRI. Being much cheaper, CESM may constitute a good alternative for improving diagnostic sensitivity in these countries. In this paper, we present a challenging case of the concurrent and co-located presentation of a fibroadenoma and a triple negative invasive carcinoma of no special type (TNBC-NST). The malignancy was indistinguishable from the fibroadenoma by mammography. By ultrasound, a suspicious area was observed and biopsied, but the histopathology did not confirm a cancer diagnosis. As the suspicion was not confirmed, a second stage of the imaging diagnosis using CESM was recommended. This technique allowed clear visualization of the malignancy, which was finally excised by breast-conserving surgery. This case reveals the potential of CESM as an easy, rapid and inexpensive new technique for the diagnosis of malignancies that might easily remain occult to mammography plus breast ultrasound (BUS).
\end{abstract}

Keywords: Radiographic Image Enhancement, Triple Negative Breast Neoplasms, Contrast Media, Developing Countries, Subtraction Technique

\section{Introduction}

Fibroadenoma is a benign lesion of the breast that is frequently reported in young women (in their twenties or early thirties), but much less commonly reported in women older than 40 years (1). This lesion is frequently associated with other benign pathologies $(1,2)$, but also has been reported as associated, or concomitant, with malignancies in up to $5 \%$ of cases, mainly in older patients (2). In extremely rare cases, these malignancies may be colocated with, or even within a fibroadenoma (0.02\%) (3). It has been reported that a high percentage of these concurrent malignancies may be missed $(1,2)$, and for this reason, radiologists should be very cautious when evaluating fibroadenomas of a given patient within this specific age group. In recent years, new imaging methods have been developed, which increase the sensitivity for the diagnosis of breast cancers using contrast media. Of these, contrastenhanced breast magnetic resonance imaging (MRI) currently appears as the most sensitive technique to detect and stage breast cancer $(4,5)$. However, several recent studies have shown that the emerging dual-energy, contrastenhanced digital mammography (CESM) method enables accurate detection of malignant breast lesions with a similar performance to that of MRI (4, 6-8), making it a good alternative to this expensive technique.

In this paper, we present a case of a complex breast lesion consisting of the co-localization of triple negative invasive carcinoma of no special type (TNBC-NST) and a fibroadenoma. The malignant lesion was mammographically indistinguishable from the fibroadenoma, and was negative on the image-guided biopsy of an only-subtly sus- 
picious area on breast ultrasound (BUS). However, the malignancy could be clearly visualized in the second stage of the imaging diagnosis, using CESM. This case is presented to reveal the potential of CESM, as an easy, rapid and inexpensive alternative to MRI for the diagnosis of malignancies, which are occult to mammography plus BUS.

\section{Case Presentation}

A 60-year-old woman underwent a screening mammogram (MX) in the breast imaging section of our hospital. She had a family history of breast cancer (mother). The patient reported no palpable masses on self-exploration. Skin retraction, nipple discharge or palpable lymph nodes were not observed on clinical examination previous to the MX. The right breast MX showed a $1.5 \mathrm{~cm}$ oval-shaped nodular mass with well-defined borders located in the upper-outer quadrant with imaging features of probable fibroadenoma. The left breast MX showed a wellcircumscribed mass of irregular shape of about $1.7 \mathrm{~cm}$, also in the upper-outer quadrant, with imaging features that were compatible with either a single irregularly shaped fibroadenoma, or with two smaller adjacent round-shaped fibroadenomas. A whole breast ultrasound (US) was recommended to further define the diagnosis of these lesions. The right breast US gave an image of $1.5 \mathrm{~cm}$, compatible with simple fibroadenoma. The left breast US showed an image compatible either with fibroadenoma or intramammary ganglion, but also showed an adjacent suspicious $0.7 \times 1 \mathrm{~cm}$ hypoechoic area with an abrupt boundary and posterior acoustic enhancement (Figure 1). We proceeded to perform an ultrasound-guided biopsy of the lesions of the right and left breasts, and the histopathological result was fibroadenoma with areas of microcalcification in all three cases. Because US suspicion of malignancy in the left breast was not confirmed (and taking into account the age of the patient), we decided to perform a dual-energy, contrast-enhanced spectral mammography (CESM). With the patient in the seated position the iodinated contrast was administered $(1.5 \mathrm{mg} / \mathrm{kg} \mathrm{IV}$, at $3 \mathrm{~mL} / \mathrm{s})$, and after two minutes the acquisition of lowenergy (28 kVp) and high-energy ( $47 \mathrm{kVp}$ ) images began in the following order: a) medial-lateral oblique view, nonsuspicious breast; b) cranial-caudal view, non-suspicious breast; c) cranial-caudal view, suspicious breast; and d) medial-lateral oblique view, suspicious breast, with a total time for the acquisition of all images of five minutes. The CESM study showed no pathological enhancement in the right breast. The low-energy image of CESM of the left breast (equivalent to conventional digital mammography) showed the same lesion with the aspect of fibroadenoma as seen in MX (Figure 2A and B), but the combined image revealed a $9.8 \mathrm{~mm}$ nodular enhancement with irregular and ill-defined contours (Figure 3), which corresponded with one of the two sections of the irregular lesion seen both in MX and in the low-energy image of CESM (Figure $2 \mathrm{C}$ and D). A US-guided implantation of a metallic marker was performed for the localization of this lesion, which was surgically excised. The histopathology revealed triplenegative invasive carcinoma of no special type (TNBC-NST), plus an independent fibroadenoma (Figure 4). It should be noted that these two lesions were completely independent of each other.

\section{Discussion}

Fibroadenoma of the breast is a very common benign lesion, especially in younger women, and is considered more closely related to an aberrant development of the breast tissue than to a true neoplasm (2). Although in general fibroadenoma is not considered to increase the risk of cancer, it is often associated to other pathologies such as sclerosing adenosis, duct ectasia, epithelial apocrine metaplasia, fibrocystic disease, and papillomatosis (1). However, in women over 40 years of age with fibroadenoma, the concomitant occurrence of invasive cancers has been reported in up to $5 \%$ of cases (2). So, the case presented here is not particularly rare, but two facts made the imaging diagnosis of this case particularly challenging: the type of tumor and its location. First, the tumor type, triple negative invasive ductal carcinoma of no special type, has been described as a tumor that often does not exhibit major suspicious findings of malignancy by mammography or US such as an irregular shape, ill-defined margins, or microcalcifications (9). In this case, in the mammogram, the tumor gave an appearance indistinguishable from a fibroadenoma. Secondly, its location was adjacent to a true fibroadenoma, and the two masses appeared partially superimposed on the mammographic image, giving the appearance of either an irregularly shaped single fibroadenoma or of two adjacent rounded and smaller fibroadenomas.

It has been reported that a small percentage of fibroadenomas in older women may harbor malignant tumors inside $(0.02 \%$ to $0.125 \%$ ) (3). However, the pathology of the surgical specimen revealed that in this case, these two lesions were completely independent, although located very close to each other. For these two reasons, this case could easily have fallen within the $4-19 \%$ of malignant breast lesions that are missed by mammography (10). Moreover, in this case, although BUS gave a suspicion of malignancy in a part of the lesion, the guided biopsy resulted in a histopathological diagnosis of fibroadenoma. Probably due to the proximity of both lesions, the biopsy 

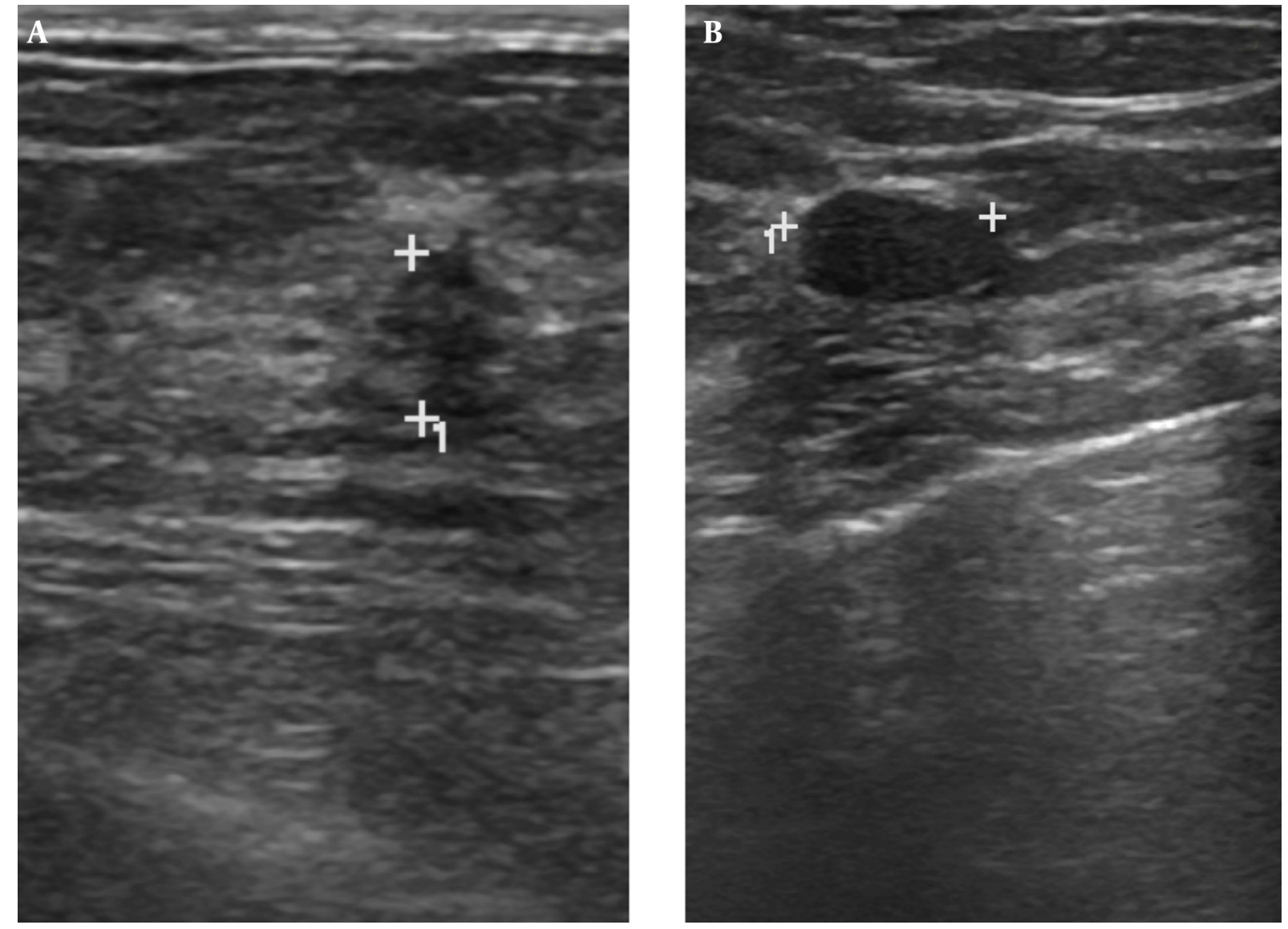

Figure 1. A 60-year-old woman with a family history of breast cancer and mass lesion in left screening mammography. Ultrasound study of the upper-outer quadrant of the left breast where two adjacent lesions are seen, A, Suspicious hypoechoic area; B, Hypoechoic solid lesion with well-defined borders with subtle posterior enhancement, which is compatible with fibroadenoma. After percutaneous US-guided biopsy, the histopathological result was fibroadenoma for both lesions.

procedure was imprecise and incorrectly took a sample of the benign lesion instead of the carcinoma. Mainly due to the age of the patient, additional imaging studies were recommended, since it has been reported that fibroadenomas may be associated with malignant lesions with some frequency in older women (2).

In the last decade, new methods have been developed to improve the sensitivity in the diagnosis of breast lesions, focusing on characteristics such as the neoangiogenesis of malignancies. Thus, techniques, such as contrastenhanced breast MRI or CESM, have been developed (4). Currently it is accepted that MRI is the most sensitive imaging technique for the diagnosis and staging of breast cancer (5). However, MRI is an expensive technique that is not yet readily available in many developing countries. In addition, MRI also presents limitations for some patients such as overweight or claustrophobic women, or patients wearing pacemakers or metallic prostheses. CESM is a recently introduced imaging modality(2011), which is based on the attenuation of radiation as it passes through different materials, in this case iodine and soft tissue. Hence, after injecting iodinated contrast the usual mammographic views are presented, with the difference being that two consecutives images are acquired for each view: one of low energy and the other one of high energy (8). Post-processing allows for the creation of one combined image, where contrast uptake-areas are enhanced, and the normal tissue areas are suppressed (8). Thus, CESM is both a routine mammographic study and a contrasted image capable of finding angiogenesis. This technique is very new, and the characterization of benign and malignant breast lesions on CESM is still a subject of research. To date, some pitfalls and disadvantages of CESM have been described such as its limited performance with malignant microcalcifications with no underlying mass $(8,11)$, with the interpretation of ganglions and adenopathies (8), or in patients with silicone 

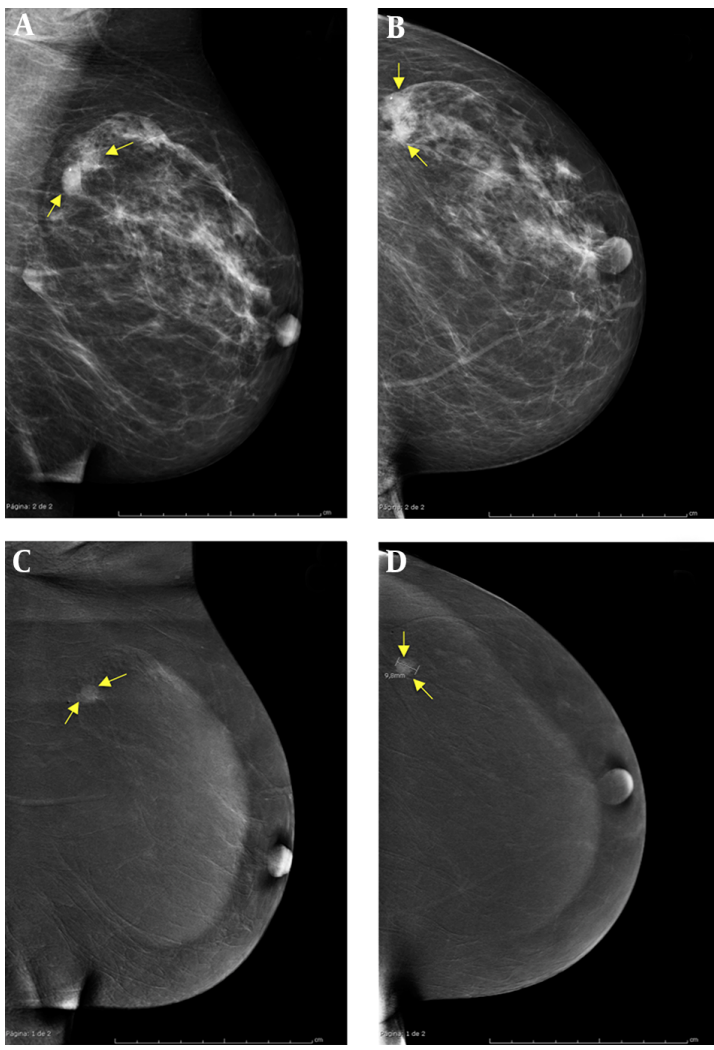

Figure 2. Dual-energy contrast-enhanced spectral mammogram of left breast. Panels of low-energy mediolateral oblique (A) and craniocaudal (B) images show a well defined lesion of $1.7 \mathrm{~cm}$, which present the features of fibroadenoma. Panels of combined mediolateral oblique (C) and craniocaudal (D) images show no enhancement of the fiboradenomatous lesion but reveal a new enhancement that remained hidden behind: triple-negative invasive carcinoma of no special type.

breast implants (8). In addition, the fact that CESM requires approximately a 1.5 times greater radiation dose than conventional mammography $(8,11)$ has been seen as a disadvantage. However, to date, the false negative and false positive rates of CESM have been reported to be very low (11), and recent studies have demonstrated that it could have a sensitivity and sensibility similar to that of MRI, while having the advantage of being faster, much more economical, and not presenting the aforementioned contraindications of MRI $(4,6,7)$. As such, CESM is a very promising imaging diagnostic tool, which may be useful in the diagnosis of complex breast lesions, as illustrated by this presented case report.

\section{Footnotes}

Authors' Contribution: Manager of the integrity of the study: Maria Del Mar Travieso Aja; original idea of the study: Maria Del Mar Travieso Aja and Octavio P. Luzardo;

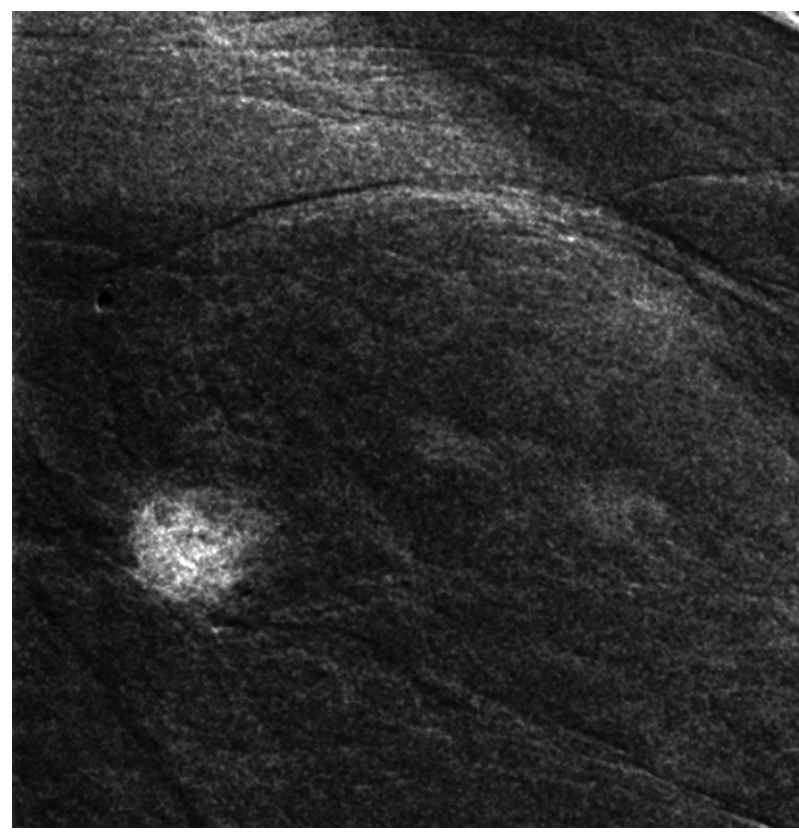

Figure 3. Dual-energy, contrast-enhanced spectral mammogram of left breast, Magnification of the contrast-enhanced lesion in the combined image showing an irregular shape and ill-defined borders.

data mining: Maria Del Mar Travieso Aja, Purificacion Munoz, Mario Rodriguez Rodriguez and Victor Vega Benitez; reference search: Maria Del Mar Travieso Aja and Octavio P. Luzardo; writing: Maria Del Mar Travieso Aja and Octavio P. Luzardo; manuscript critical review with intellectually relevant contributions: Maria Del Mar Travieso Aja, Octavio P. Luzardo, Purificacion Munoz, Mario Rodriguez Rodriguez and Victor Vega Benitez; final version approval: Maria Del Mar Travieso Aja, Octavio P. Luzardo, Purificacion Munoz, Mario Rodriguez Rodriguez and Victor Vega Benitez.

Financial Disclosure: The authors have no financial interests related to the material in this manuscript.

Funding/Support: No funding support was provided to the authors of this study. 

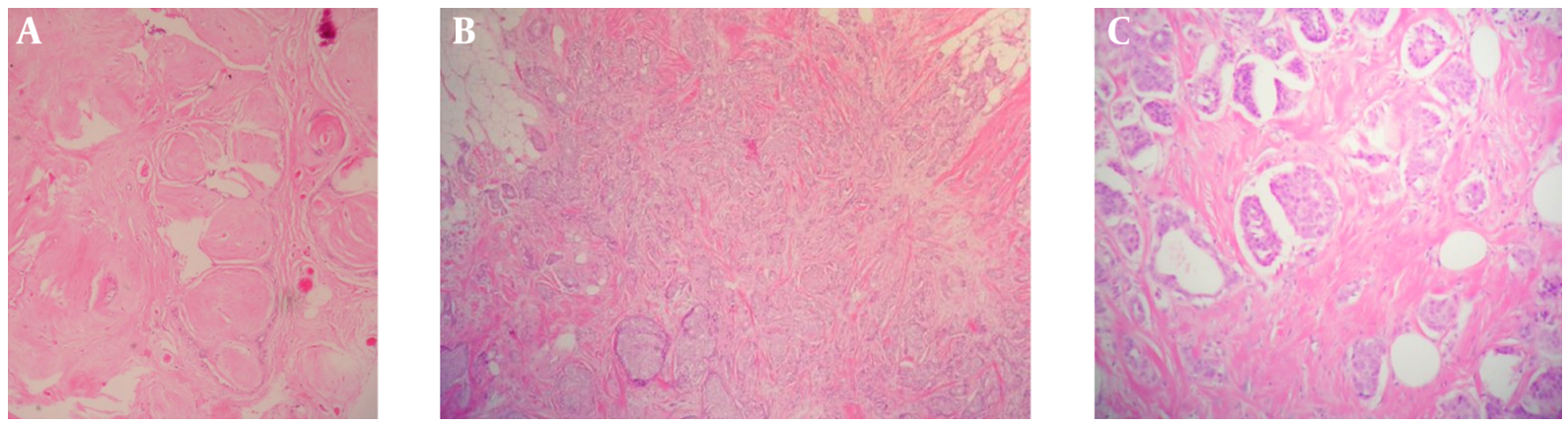

Figure 4. Histology of the lesions; A, Partially sclerotic fibroadenoma, (hematoxylin and eosin, $100 \times$ ); B, Triple negative infiltrating carcinoma of no special type (hematoxylin and eosin, $40 \times$ ); C, Magnification of the TNBC-NST (hematoxylin and eosin, $200 \times$ ).

\section{References}

1. Amin AL, Purdy AC, Mattingly JD, Kong AL, Termuhlen PM. Benign breast disease. Surg Clin North Am. 2013;93(2):299-308. doi: 10.1016/j.suc.2013.01.001. [PubMed: 23464687].

2. Shabtai M, Saavedra-Malinger P, Shabtai EL, Rosin D, Kuriansky J, Ravid-Megido M, et al. Fibroadenoma of the breast: analysis of associated pathological entities-a different risk marker in different age groups for concurrent breast cancer. Isr Med Assoc J. 2001;3(11):813-7. [PubMed: 11729575].

3. Wu YT, Chen ST, Chen CJ, Kuo YL, Tseng LM, Chen DR, et al. Breast cancer arising within fibroadenoma: collective analysis of case reports in the literature and hints on treatment policy. World J Surg Oncol. 2014;12:335. doi: 10.1186/1477-7819-12-335. [PubMed: 25382741].

4. Fallenberg EM, Dromain C, Diekmann F, Engelken F, Krohn M, Singh JM, et al. Contrast-enhanced spectral mammography versus MRI: Initial results in the detection of breast cancer and assessment of tumour size. Eur Radiol. 2014;24(1):256-64. doi: 10.1007/s00330-0133007-7. [PubMed: 24048724].

5. Argus A, Mahoney MC. Indications for breast MRI: case-based review. AJR Am J Roentgenol. 2011;196(3 Suppl):WS1-14. doi: 10.2214/AJR.09.7213. [PubMed: 21343536].
6. Lobbes MB, Lalji UC, Nelemans PJ, Houben I, Smidt ML, Heuts E, et al. The quality of tumor size assessment by contrast-enhanced spectral mammography and the benefit of additional breast MRI. J Cancer. 2015;6(2):144-50. doi: 10.7150/jca.10705. [PubMed: 25561979].

7. Thibault F, Balleyguier C, Tardivon A, Dromain C. Contrast enhanced spectral mammography: better than MRI?.EurJ Radiol. 2012;81 Suppl 1:S162-4. doi: 10.1016/s0720-048x(12)70068-2. [PubMed: 23083575].

8. Travieso Aja MM, Rodriguez Rodriguez M, Alayon Hernandez S, Vega Benitez V, Luzardo OP. Dual-energy contrast-enhanced mammography. Radiologia. 2014;56(5):390-9. doi: 10.1016/j.rx.2014.05.003. [PubMed: 25086679].

9. Jung HK, Han K, Lee YJ, Moon HJ, Kim EK, Kim MJ. Mammographic and sonographic features of triple-negative invasive carcinoma of no special type. Ultrasound Med Biol. 2015;41(2):375-83. doi: 10.1016/j.ultrasmedbio.2014.09.006. [PubMed: 25542493].

10. Holland R, Hendriks JH, Mravunac M. Mammographically occult breast cancer. A pathologic and radiologic study. Cancer. 1983;52(10):1810-9. [PubMed: 6313174].

11. Dromain C, Thibault F, Diekmann F, Fallenberg EM, Jong RA, Koomen $\mathrm{M}$, et al. Dual-energy contrast-enhanced digital mammography: initial clinical results of a multireader, multicase study. Breast CancerRes. 2012;14(3):R94. doi: 10.1186/bcr3210. [PubMed: 22697607]. 